\title{
Biomimetic and electroactive 3D scaffolds for human neural crest-derived stem cell expansion and osteogenic differentiation
}

\begin{abstract}
Donata landolo (D), Department of Chemical Engineering and Biotechnology, University of Cambridge, Philippa Fawcett Drive, Cambridge CB30AS, UK Jonathan Sheard, Stem Cell Biology and Regenerative Medicine Group, School of Pharmacy, University of Reading, Whiteknights Campus, Reading RG66AP, UK; Sheard BioTech Limited, Wenlock Road, London N17GU, UK

Galit Karavitas Levy, Department of Engineering, University of Cambridge, Trumpington Street, Cambridge CB21PZ, UK

Charalampos Pitsalidis, Department of Chemical Engineering and Biotechnology, University of Cambridge, Philippa Fawcett Drive, Cambridge CB30AS, UK

Ellasia Tan, Department of Physics and Centre for Plastic Electronics, Imperial College London, South Kensington, UKSW7 2B London, UK

Anthony Dennis (D, Department of Engineering, University of Cambridge, Trumpington Street, Cambridge CB21PZ, UK

Ji-Seon Kim, Department of Physics and Centre for Plastic Electronics, Imperial College London, South Kensington, UKSW7 2B London, UK

Athina E. Markaki, Department of Engineering, University of Cambridge, Trumpington Street, Cambridge CB21PZ, UK

Darius Widera (1), Stem Cell Biology and Regenerative Medicine Group, School of Pharmacy, University of Reading, Whiteknights Campus, Reading RG66AP, UK

Róisín M. Owens (DD, Department of Chemical Engineering and Biotechnology, University of Cambridge, Philippa Fawcett Drive, Cambridge CB30AS, UK
\end{abstract}

Address all correspondence to Donata landolo at donata.iandolo@emse.fr

(Received 27 November 2019; accepted 10 January 2020)

\section{Abstract}

Osteoporosis is a skeletal disease characterized by bone loss and bone microarchitectural deterioration. The combination of smart materials and stem cells represents a new therapeutic approach. In the present study, highly porous scaffolds are prepared by combining the conducting polymer PEDOT:PSS with collagen type I, the most abundant protein in bone. The inclusion of collagen proves to be an effective way to modulate their mechanical properties and it induces an increase in scaffolds' electrochemical impedance. The biomimetic scaffolds support neural crest-derived stem cell osteogenic differentiation, with no need for scaffold pre-conditioning contrarily to other reports.

\section{Introduction}

Osteoporosis affects more than 75 million patients in the EU, USA, and Japan with increasing prevalence correlating with the rising life expectancy. It is expected that the number of patients affected by these disorders will increase by a third by 2050 . ${ }^{[1]}$ One of the recent innovative approaches to tackle osteoporosis entails the use of autologous stem cells in combination with a material that can actively influence their behavior. In this context, autologous mesenchymal stem cells (MSCs) able to generate osteoblasts have been proposed to be good candidates to treat osteoporosis. ${ }^{[2]}$ Recently, it has been shown that the application of MSCs can prevent bone loss in a mouse model of osteoporosis. ${ }^{[3]}$ However, osteoporosis results in decreased numbers of MSCs with low osteogenic potential. ${ }^{[4-6]}$ Thus, alternative cell sources for the treatment of age-related bone disorders are needed. In this context, craniofacial neural crestderived stem cells (NCSCs) have been shown to be nonimmunogenic $^{[7]}$ and to possess osteogenic differentiation potential similar to MSCs. ${ }^{[8]}$ In particular, adult post-migratory NCSCs can be efficiently isolated from various tissue types and

\footnotetext{
† Present address: Mines Saint-Etienne, University of Lyon, Université Jean Monnet, UMR INSERM U1059, 158 Cours Fauriel, Saint-Etienne 42023, France.
}

undergo osteogenic differentiation in 2D and 3D environments with an efficacy comparable to MSCs. ${ }^{[9-13]}$ In order to develop a targeted regenerative approach to restore lost bone and to help the body recover the lost function, cells have been employed together with materials and biomaterials. A number of substrates have been developed using a large variety of materials (i.e., natural, synthetic, metal, nanoparticle-based materials). ${ }^{[14,15]}$ Several biomimetic substrates are available that can recapitulate the biochemical and biophysical properties of the target tissue and the microenvironment experienced by cells. Important biophysical factors influencing cell behavior in the body include not only surface morphology and mechanical properties but also the bioelectricity. Different tissues are indeed characterized by diverse morphological and mechanical properties and a different ability to respond to a local change of the electrical field they experience. One such tissue is bone, as it was shown to display both piezoelectricity and flexoelectricity. ${ }^{[16-18]}$ This ability to generate electricity is key for bone regeneration and has therefore inspired the use of electrical cues to develop alternative strategies towards effective bone tissue engineering strategies. In order to investigate and to harness cell and tissue bioelectricity, conducting polymers (CPs) have been successfully used to develop smart, electroactive scaffolds. Due to their easy processability, $\mathrm{CPs}$, such as polyaniline 
(PANI), polypyrrole (PPy), and polythiophene and their derivatives and composites have been adopted using different techniques to develop 2D and 3D substrates. ${ }^{[19,20]}$ CPs have several further advantageous properties including good cyto- and biocompatibility, facile synthesis, and simple modification. CPs have the advantage over traditional silicon-based electronics to bridge the mechanical mismatch with the target tissue. Among other CPs, poly(3,4-ethylenedioxythiophene) polystyrene sulfonate (PEDOT:PSS) has been adopted for different applications targeting cells in vitro and in vivo. ${ }^{[21-23]}$ PEDOT:PSS has the potential to be processed with a number of techniques allowing for adjusting its chemistry and its intrinsic mechanical properties, and it has the ability to conduct both ionic and electronic currents. ${ }^{[24]}$ A diverse range of devices have been generated based on this $\mathrm{CP}$, having different geometry, mechanical properties, and target function. ${ }^{[21-27]}$ Given this mixed conductivity, PEDOT:PSS has been used widely both in sensing and delivery of electric cues. This feature is indeed key for developing a material able to simultaneously host and guide stem cell differentiation and to monitor the cell fate and status. ${ }^{[22,28]}$ Previous experiments have shown that it is possible to use PEDOT:PSS for cell proliferation and monitoring in both $2 \mathrm{D}$ and $3 \mathrm{D}$ environments. ${ }^{[22,28-32]}$ In this study, the biomimicry potential of the polymer is enhanced by introducing collagen type I, the major protein component of the bone extracellular matrix. ${ }^{[33,34]}$ Collagen, like other biopolymers including glycosaminoglycans, gelatin, chitosan, silk fibrin, and elastin, has the important characteristic of presenting chemical groups that are recognized by cells and to which cells can bind. ${ }^{[35]}$ In particular, collagen plays an important role in facilitating the differentiation of bone progenitor cells into osteoblasts by interacting with the cell cytoskeleton and inducing, among other processes, cell proliferation. ${ }^{[36,37]}$ The materials developed here harness the properties of both the PEDOT:PSS and collagen components, leading to a highly biomimetic, electroactive scaffold for stem cell expansion and differentiation. The use of CPs at the interphase with stem cells has been explored for a number of applications. ${ }^{[38,39]}$ Osteogenic differentiation of stem cells has been previously demonstrated by different groups, but the cells mostly used are MSCss. ${ }^{[23,40-42]}$ Here we investigate, for the first time, the response of NCSC to a $3 \mathrm{D}$ electroactive and biomimetic environment for bone tissue engineering. The surface, electrochemical, as well as chemical characterization of the macroporous electroactive scaffolds, is shown, together with the assessment of their cytocompatibility. The differential response of NCSC to scaffold composition is evaluated in terms of their ability to differentiate towards bone-forming cells.

\section{Results and discussion Scaffold fabrication}

We developed electroactive, biomimetic scaffolds that could be used to culture stem cells and induce their osteogenic differentiation. The biomimetic scaffolds are based on the commercially available, CP PEDOT:PSS. Collagen type I was added in order to enhance the biomimicry potential of the surfaces. Collagen I was selected as it is the main protein component of the bone matrix. In this study, we investigated not only the presence but also the level of collagen in terms of its effect on both the scaffold properties (morphological, rheological, electrical, and mechanical) and the cell behavior. The process of ice-templating (freeze-drying) was adopted to create highly porous scaffolds with pore sizes compatible with cell infiltration and proliferation. The freeze-drying process parameters were adapted from previously developed processes for similar materials. ${ }^{[43]}$ In order to investigate the effect of the absence, presence, and level of collagen type 1 on cell behavior, different ratios of collagen type 1 to PEDOT:PSS were explored. Two ratios of collagen to PEDOT:PSS were investigated corresponding to weight ratios of $0.3 \%$ and $1.1 \%(\mathrm{w} / \mathrm{w})$ and they were compared to the pristine scaffolds containing no collagen. Throughout this work, they will be referred to as P:P, COLL1 $(0.3 \mathrm{wt} \%)$ and COLL3 (1.1. wt $\%)$, respectively. To ensure homogenous distribution of the protein in the highly acidic PEDOT:PSS dispersion, blends were sonicated in a water bath to which ice was added to avoid local heating (Fig. 1). Once the dispersions were homogenous and no aggregates were found, the solution was poured in the selected molds and the freeze-drying process started. When the process was over, samples were exposed to thermal treatment in order to accelerate crosslinking and therefore guarantee their prolonged water stability. The morphology of the obtained scaffolds is similar to that of PEDOT:PSS-based scaffolds previously reported. ${ }^{[22,43]}$

\section{Scaffold morphology and pore characterization}

Highly porous structures were obtained and analyzed by scanning electron microscope (SEM) [Fig. 1, Supplementary Fig. S1(a)]. The scaffold pore size was calculated using the SEM images. Increasing the collagen content did not seem to affect the dimensions of the pores formed during the freezedrying process [Fig. 2(a)]. Pristine PEDOT:PSS scaffolds were shown to have average pore diameters of $180 \pm 39 \mu \mathrm{m}$, whereas COLL1 and COLL3 samples had pores of $141 \pm 34$ $\mu \mathrm{m}$ and $118 \pm 19 \mu \mathrm{m}$. There was no statistically significant difference in pore sizes between the samples with the two collagen concentrations, but the inclusion of collagen did prove to have an effect on pore dimensions falling in the right range to allow cell adhesion, penetration and nutrient, oxygen exchange, and waste removal. ${ }^{[4,45]}$ Murphy and colleagues showed that larger pores $(325 \mu \mathrm{m})$ favor cell proliferation rather than cell attachment. Scaffold porosity was also calculated analyzing SEM images. Porosity decreased significantly when collagen was included in the blends, decreasing from $40.7 \pm 2.9 \%$ for pristine scaffolds, to $33.2 \pm 2.3 \%$ for COLL1 and $25.8 \pm 3.8 \%$ for COLL3. Pore interconnectivity was also investigated using micro computed tomography, revealing a highly interconnected network of pores (Supplementary Video S1). Highly porous materials, displaying highly interconnected pores, are key for 

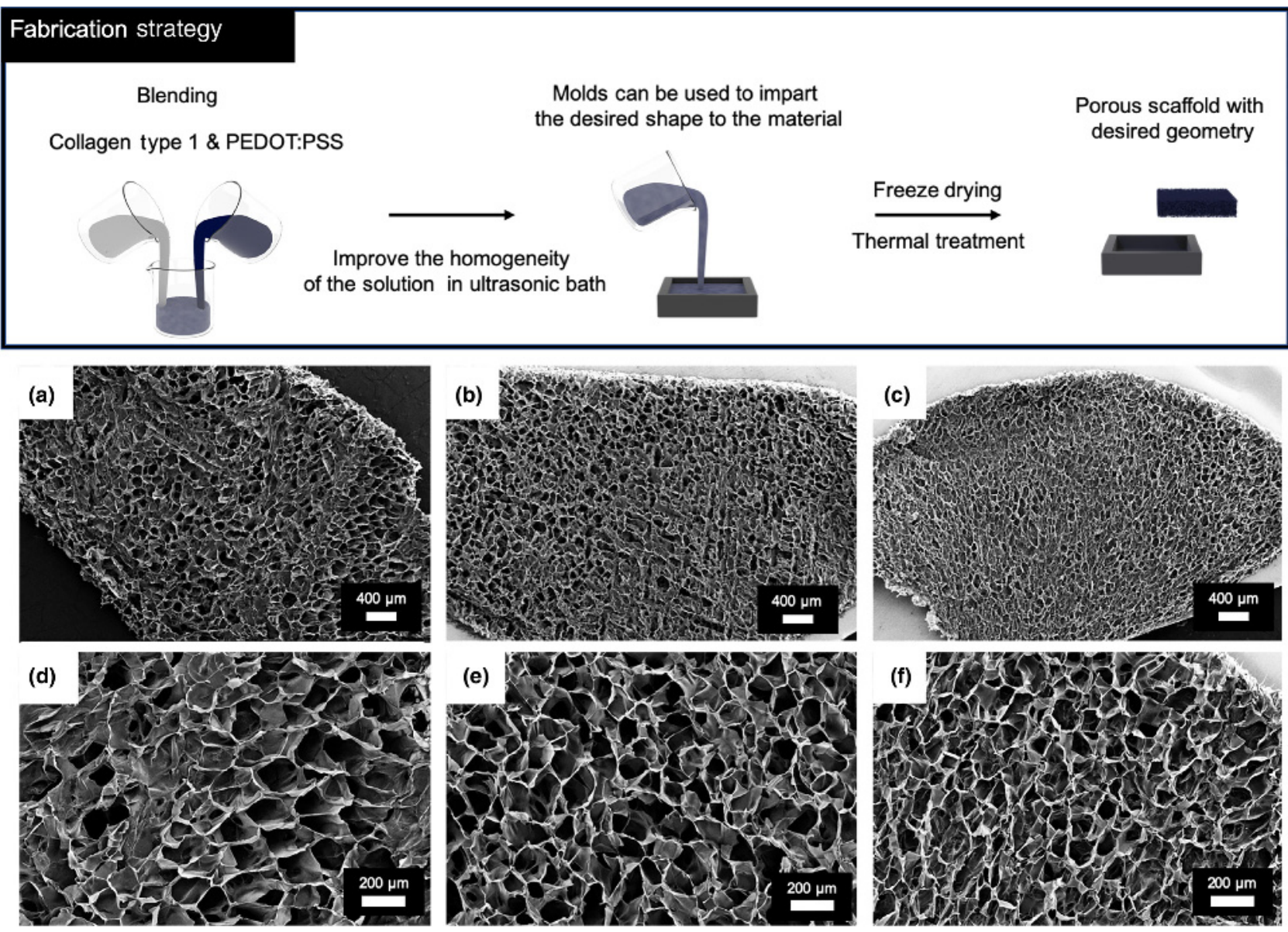

Figure 1. Schematic of the production and surface characterization of the porous scaffolds. Top panel: the scheme reproduces the different steps from the preparation of the blends to the freeze-drying and thermal treatment (schematic by http://conceptualized.tech). Bottom panels: Scaffold surface morphology. (a-f) SEM images of P:P, COLL1, and COLL3 scaffolds at increasing magnifications. (a-c) shows how the overall surface of the scaffolds appear after the fabrication process and slices have been obtained with the vibrating blade microtome. Scale bar: $400 \mu \mathrm{m}$. (d-f) shows, with greater detail, the distribution of pores and their structure. Scale bar: $200 \mu \mathrm{m}$.

cell material colonization, migration, and for an appropriate exchange of media, cellular waste, and gases $\left(\mathrm{CO}_{2}\right.$ and $\left.\mathrm{O}_{2}\right)$.

\section{Compressive Young's modulus of the scaffolds}

It is well known that the scaffold stiffness affects stem cell differentiation ${ }^{[46]}$ and the load transfer to the attached cells when the scaffold deforms under physiological conditions. Young's modulus of the scaffolds investigated here was measured using a technique customized for low stiffness structures ${ }^{[47]}$. A customized "see-saw" set-up, with a lower inertia compared to a standard Instron machine, was used to measure the compressive Young's modulus of the scaffolds. Young's modulus values were obtained from the tangent of the loading curves giving $90.8 \pm 9.5 \mathrm{kPa}$ for the bare $\mathrm{P}: \mathrm{P}$ scaffolds and $36.3 \pm$ 3.8 and $33.5 \pm 0.9 \mathrm{kPa}$ for the COLL1 and COLL3 scaffolds, respectively [Fig. 2(b)]. While it is difficult to directly compare the scaffolds since they have different pore sizes and contents [Fig. 2(a) and Supplementary Fig. S1(b)], the measurements suggest that the P:P scaffolds, with the higher porosity and average pore sizes, have a stiffer cell wall material compared to the COLL1 and COLL3 scaffolds. There was no statistically significant difference in Young's modulus between the COLL1 and COLL3 scaffolds. While increasing the collagen content from 0.3 to $1.1 \%(\mathrm{w} / \mathrm{w})$ presumably reduces the stiffness of the cell wall material (as the P:P concentration is reduced), this increase is probably offset by the lower porosity of the COLL3 scaffolds. Interestingly, researchers measured the elastic properties of the osteoid (the highly crosslinked collagen network secreted by osteoblasts in which MSCs undergo a transition to preosteoblasts, that will be transformed into bone) and found that its mechanical properties are approximately $27 \pm 10 \mathrm{kPa}^{[46]}$ These values nicely match with those reported in this work, highlighting the biomimicry potential of the developed scaffolds.

\section{Chemical characterization of the scaffolds}

To assess the presence and distribution of collagen, Raman spectroscopy measurements were run on cross-sections of the porous scaffolds. Raman signals specific to collagen are located 

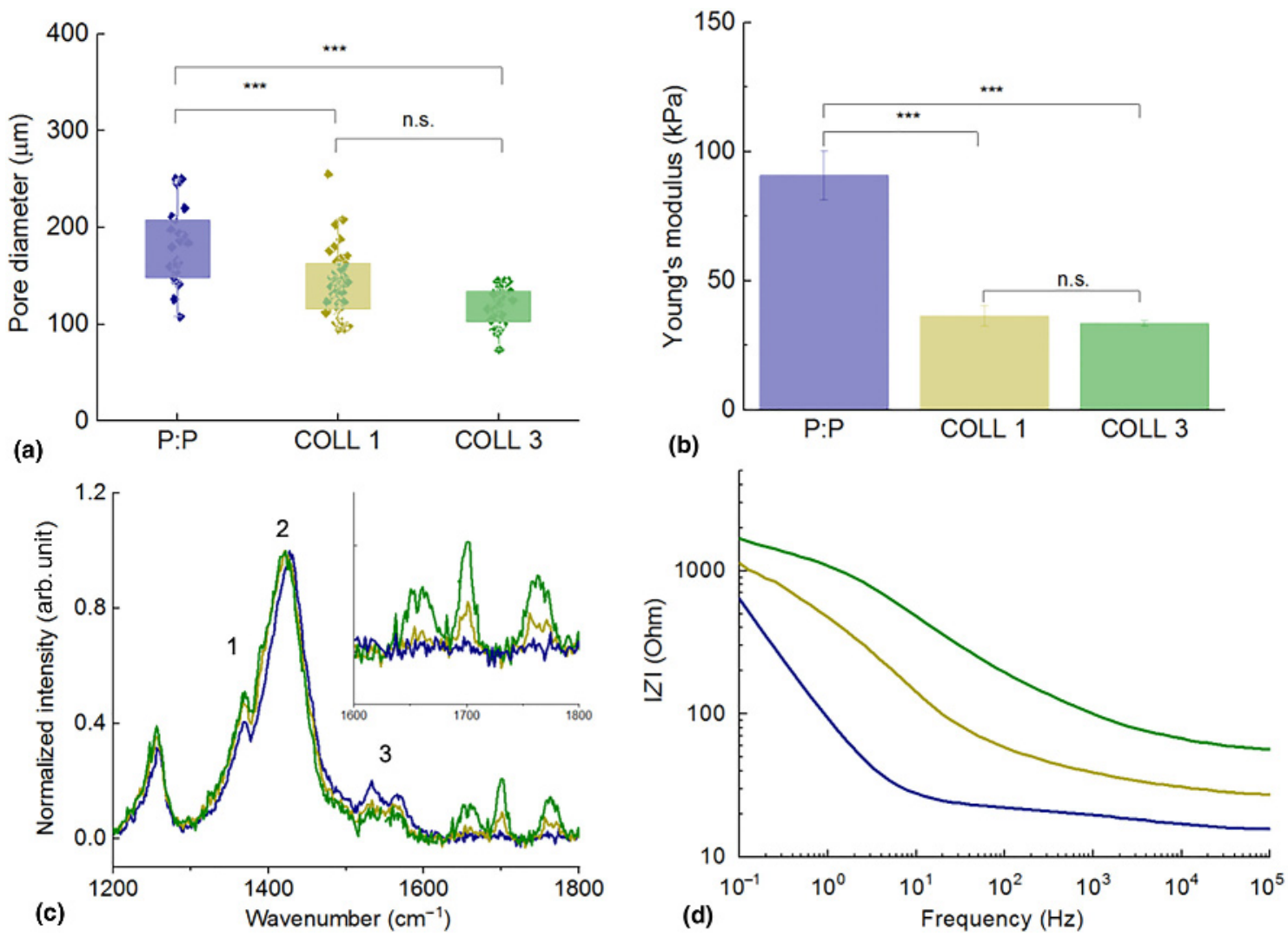

Figure 2. Characterization of scaffold properties. (a) Pore-size distribution for the three scaffolds. ${ }^{* \star \star} P<0.001$, n.s.: the difference is not statistically significant. (b) Through-thickness Young's modulus of the three scaffolds. ${ }^{* *}{ }^{*} P<0.001$, n.s.: the difference is not statistically significant. (c) Raman spectrum corresponding to the area including the three main vibrational modes of PEDOT:PSS [intra-ring C-C (1), symmetric (2), and asymmetric (3) $C=C$ ]. The inset shows the area of the spectrum containing the collagen-specific peaks. (d) Bode plot of the impedance of the PEDOT:PSS scaffolds with the three compositions, respectively. P:P pristine: blue, COLL1: light green, COLL3: dark green.

at 1652,1700 , and $1758 \mathrm{~cm}^{-1}$ [inset Fig. 2(c)]. Particularly, the $1652 \mathrm{~cm}^{-1}$ peak is related to the amide(I) $\mathrm{C}=\mathrm{O}$ vibrational mode. ${ }^{[48,49]}$ The position and intensity of the peaks characteristic for PEDOT:PSS were determined for all the scaffold compositions under analysis [Fig. 2(c)]. The main differences were at the three main vibrational modes of PEDOT:PSS [namely, intra-ring $\mathrm{C}-\mathrm{C}$ (1), symmetric (2), and asymmetric (3) $\mathrm{C}=\mathrm{C}$ ] [Fig. 3(c)]. ${ }^{[50,51]}$ There is a shift of the $\mathrm{C}=\mathrm{C}$ symmetric mode (peak 2) to lower vibrational energy, which is caused by subtle changes in the contribution of the neutral and doped PEDOT conformations to this peak. ${ }^{[52,53]}$ Therefore, the Raman peak shift towards lower wavenumbers is characteristic of an increase in the neutral PEDOT conformation connected to a dedoping effect on the PEDOT:PSS in the scaffold. ${ }^{[54]}$ The collagen could have induced molecular packing changes in PEDOT:PSS. PEDOT:PSS is a conjugated CP made of a highly interconnected network of polymeric chains, where the positive charges distributed along the PEDOT backbone are compensated by the negative charges carried by PSS, due to the presence of the sulfonic acid moieties. The overall $\mathrm{pH}$ of the commercial PEDOT:PSS solution is about 2, lower than the isoelectric point of collagen (Isoelectric point $\sim 8.26$ ). ${ }^{[55,56]}$
This means that when collagen is added to the blend, the protein chains will be positively charged and this could in turn lead to either the interaction of the positively charged collagen amino acids with polystyrene sulfonic acid groups or to the formation of protein aggregates. Examples of aggregates were confirmed by both SEM images and Raman spectra, acquired for extended volumes of the samples (Supplementary Fig. S2). This will therefore require further improvement of the blending protocol in the future. However, collagen also interacts with PSS and by doing so induces a restructuring of the CP. This is also reflected by the changes in the relative intensities of the peaks 1 and 3 to the main $\mathrm{C}=\mathrm{C}$ symmetric Raman peak, which indicate that there is a shift in the $\pi$-electron density of the intra-ring $\mathrm{C}-\mathrm{C}$ and $\mathrm{C}=\mathrm{C}$ bonds. Also, by binding to PSS, collagen is actually contributing to dedoping PEDOT segments. This implies an alteration in the material conductivity as we will demonstrate in the electrochemical impedance characterization.

\section{Electrochemical characterization}

Impedance measurements were run to analyze the effect of increasing concentration of collagen on the overall 

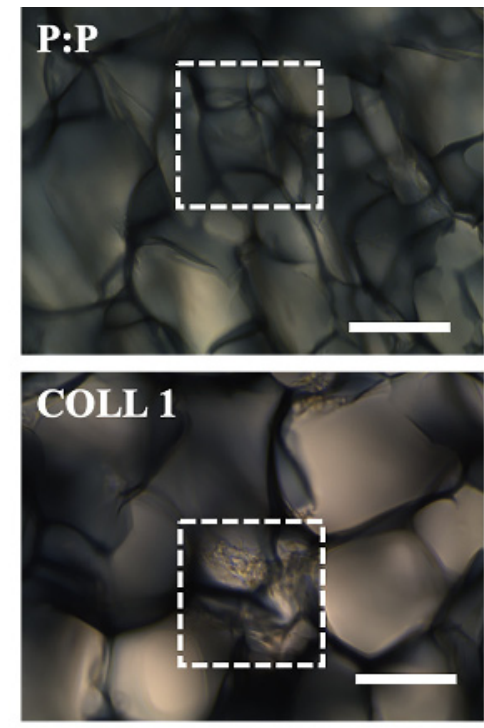

(a)

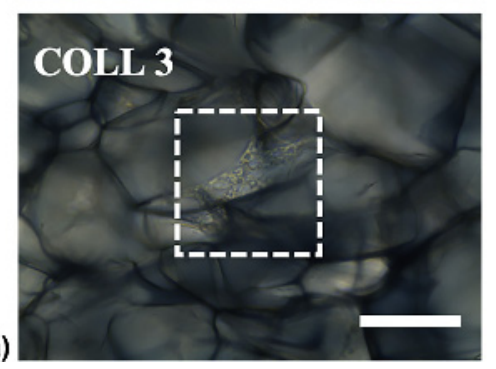

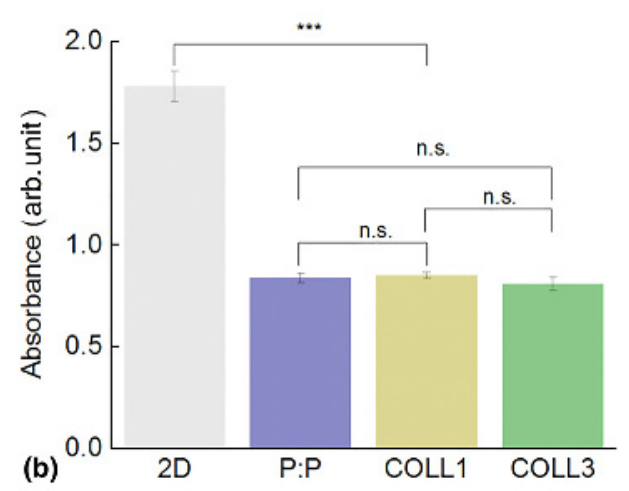

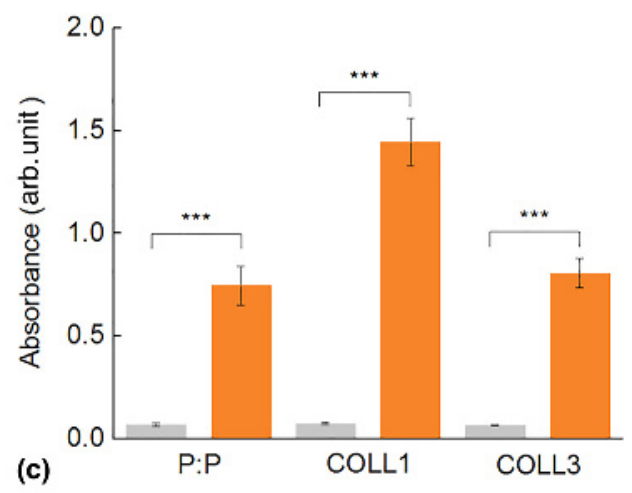

Figure 3. Scaffold cytocompatibility and osteoconductivity. (a) Brightfield images of NCSC growing on the three PEDOT:PSS-based scaffolds. Dotted squares highlight areas colonized by cells. Scale bar: $100 \mu \mathrm{m}$. (b) Viability of NCSCs measured by XTT assays. Absorbance of the three samples (P:P pristine: blue, COLL1: light green, COLL3: dark green) compared to the 2D control (gray bar). (c) Osteogenic differentiation of NCSCs. Quantitative evaluation of Alizarin red dye recovered from scaffolds after 21 days of cell culture. Gray bar: control sample in culture medium, red bars: osteogenic medium. Difference among samples was evaluated using ANOVA with ${ }^{* *} P<0.001$.

electrochemical properties of the scaffold. As anticipated, the inclusion of collagen is found to be associated with an increase in the material impedance over the whole-frequency range, as shown in the Bode and Nyquist plots [Fig. 2(d) and Supplementary Fig. S3(a)]. Specifically, the collagencontaining scaffolds exhibited a deviation from the ohmic flat curve, which is apparent in the pristine scaffolds at the mid/ high frequencies. This effect is more significant in high collagen content scaffolds (COLL3). The apparent changes were accompanied by the presence of a broad peak in the phase spectrum [Supplementary Fig. S3(b)] and a decrease in the phase angle maximum with the collagen ratio (from $\sim 45^{\circ}$ and $\sim 35^{\circ}$, respectively). Overall, however, the collagen-containing scaffolds were found to maintain their conducting characteristics, holding a great promise for using these structures in cell-based biosensing and stimulation applications.

\section{Scaffold cytocompatibility and osteoconductivity}

Neural crest-derived stem cells (NCSCs) have an enormous potential for personalized medicine as they display the remarkable characteristic of preserving their potential to differentiate towards different lineages even when isolated from adult patients. ${ }^{[9,57]}$ Their use in tissue regeneration, and more specifically for bone regeneration, could be a powerful alternative to allogenic stem cell transplantations, thus reducing the risk of rejections and the need for immunosuppression. Moreover, these populations of cells preserve their stemness also in the adult, unveiling an extremely high potential for clinical translation. ${ }^{[58,59]}$ Cells adhered and colonized the scaffolds without the need for their functionalization [Fig. 3(a)]. Cells adhered to the substrates and proliferated with no significant difference dictated by the scaffold composition. Cells were monitored in the first days of their interaction with the scaffolds by staining them with PKH67, a fluorescent dye binding to the cell membrane ( Supplementary Video S2). Cells were shown to actively explore the surfaces of the material pores before they colonize a specific area, where they adhered and proliferated. We then investigated the cytocompatibility of the scaffold by seeding NCSCs on the three different substrates and compared the cell viability to that of cells cultured on 2D surfaces after $48 \mathrm{~h}$ [Figs. 3(a) and 3(b)]. 
NCSC were tested for their ability to differentiate into boneforming cells when colonizing the PEDOT-based scaffolds. Cells were cultured in osteoinductive conditions using a commercially available medium. Interestingly, cells cultured on the COLL1 scaffold displayed higher levels of alizarin red staining and therefore of mineral matrix deposition when compared to the othe compositions. This would suggest a cooperative interplay between the scaffold composition and mechanical properties together with the external osteoinductive culture conditions and the osteoinductive properties of collagen type I. ${ }^{[13]}$ In contrast to other studies, no prior conditioning of the scaffolds was required in order to boost osteogenic differentiation. ${ }^{[31,40]}$ Interestingly, it has been demonstrated that naive MSCs exposed to substrates with increasing stiffness commit to different lineages. ${ }^{[46,60]}$ These results align with our results although the presence of an electroactive surface together with the different compositions of the substrate might introduce further variables in determining cell fate.

\section{Conclusions}

We developed highly porous, electroactive scaffolds for stem cell culture and osteogenic differentiation. Scaffold biomimicry potential was enhanced by including collagen, the major organic component of the bone matrix. The structure, Young's modulus, and electrical properties of the scaffolds were characterized. As expected, the impedance of the scaffolds is affected by the presence of collagen, due to its insulating nature. Introducing collagen not only affects scaffold conductivity but also changes its mechanical properties. Therefore, this strategy of controlling the scaffold mechanical properties would be highly beneficial to understand the influence of single factors on cell behavior in a 3D. The interplay between scaffold porosity, mechanical properties, and conductivity might dictate stem cell response to its environment. Further tests will be needed to better understand how these results correlate with scaffold properties, and more in detail which parameter of the scaffold is directing the cell behavior.

\section{Experimental section Scaffold fabrication}

Macroporous scaffolds were prepared following a slightly modified version of the procedure as reported in previous studies. ${ }^{[22,32]}$ Briefly, scaffolds were fabricated by the ice-templating technique, using an aqueous dispersion of PEDOT:PSS (PH1000, Heraeus LTD) to which 3\% (v/v) (3-glycidyloxypropyl) trimethoxysilane (GOPS, Sigma-Aldrich) was added as a crosslinker to improve scaffold stability in aqueous environments, and $0.25 \%(\mathrm{v} / \mathrm{v})$ dodecyl benzenesulfonic acid (DBSA, Sigma-Aldrich) was introduced to increase scaffold electrical conductivity. In order to study the effect of the presence of collagen on cell behavior, different blends of PEDOT: PSS and Collagen I (Collagen I Rat Tail, ThermoFisher Scientific, Dreieich, Germany) were prepared having an increasing protein content $(0-1.1 \mathrm{wt} \%)$. By using a freeze-dryer (Pilote de Paillasse, Cryotec, Saint-Gély-du-Fesc, France), liquid blends were frozen down to $-50^{\circ} \mathrm{C}$ at $0.35^{\circ} \mathrm{C} / \mathrm{min}$. Ice crystals were then sublimed by ramping up the temperature at $0.12^{\circ} \mathrm{C} / \mathrm{min}$ to $20^{\circ} \mathrm{C}$. The scaffolds obtained were then thermally treated at $60^{\circ} \mathrm{C}$ for $3 \mathrm{~h}$ to let the crosslinker reticulate. $400-\mu \mathrm{m}$ thick slices were then prepared for cell culture using a VT1200 vibrating blade microtome (LEICA). Prior to cell seeding, slices were sterilized by immersion in $70 \%$ ethanol for $1 \mathrm{~h}$ followed by extensive rinsing with deionized (DI) water and phosphate buffer saline (PBS).

\section{Scaffold morphology and pore characterization}

The scaffold structure was examined using a MEB Ultra 55-Carl ZEISS scanning electron microscope (SEM). Prior to the analysis, samples were coated with $10 \mathrm{~nm}$ Gold/ Palladium and images were acquired in secondary mode using an acceleration voltage of $5 \mathrm{kV}$. Multiple images were taken for each sample in order to determine pore size and distribution. About 30-40 pores per sample were measured in order to calculate average pore sizes and pore-size distribution. Scaffold porosity was calculated using ImageJ. ${ }^{[61]}$

\section{Mechanical characterization}

The through-thickness Young's modulus of the scaffolds was measured under compression using a customized "see-saw" set-up, previously developed to test freeze-dried collagen scaffolds. ${ }^{[47]}$ Cylindrical samples of dimensions $9 \mathrm{~mm}$ (height) $\times$ $10 \mathrm{~mm}$ (diameter) were used. An increasing number of $1 \mathrm{~g}$ weights was applied at a constant rate of $10 \mathrm{~N} / \mathrm{s}$, corresponding to average stresses of around $1.4 \mathrm{kPa}$. The resulting displacement was monitored using a Keyence ${ }^{\circledR}$ bi-axial laser micrometer. Young's modulus was measured from the tangent of the loading curves. At least, five samples from each condition were tested, from which the average value was taken and the standard deviation was calculated.

\section{Raman spectroscopy}

The scaffolds were cut into smaller discs and measured using Raman spectroscopy acquired from Renishaw inVia Raman Microscope in a backscattering configuration. All measurements were performed with samples placed inside a Linkam THMS600 stage under continuous nitrogen purging to prevent any laser degradation. The calibration of the filter and grating were performed using a $\mathrm{Si}$ reference peak at $520 \mathrm{~cm}^{-1}$. The excitation source at $633 \mathrm{~nm}$ was produced by a HeNe laser and laser intensity of $10 \%$ of $12 \mathrm{~mW}$ was used with three accumulations under $50 \times$ magnification. The Raster 2D mapping was measured at each 5 and $10 \mu \mathrm{m}$ step within a filled square of $60 \mu \mathrm{m}$ across $x$ - and $y$-axis.

\section{Electrical characterization}

Impedance spectra of the scaffolds were acquired using a potentiostat (Autolab, Metrohm, Herisau, Switzerland) equipped with a frequency response analysis module. The $\mathrm{CP}$ scaffold was designated as the working electrode through the 
attachment of a gold-plated Kapton $\left({ }^{\circledR}\right.$ strip while a reticulated (vitreous) glassy carbon was used as the counter electrode. The electrolyte solution was Dulbecco's phosphate-buffered saline (PBS). The impedance $(Z)$ of the electrodes was evaluated using a two-electrode system configuration. AC voltages of frequencies ranging from 0.1 to $10^{5} \mathrm{~Hz}$ were applied and the response was measured using the impedance analyzer.

\section{Cell culture and differentiation}

Human palatal NCSCs were kindly provided by Prof. Sema Hakki, Selcuk University Faculty of Dentistry Department of Periodontology Campus, 42079 Konya, Turkey. Briefly, NCSCs were obtained from adult donors with written informed consent, and the study was approved by Ethics Committee of Dental Faculty of Selcuk University (approval number 2012-08). NCSCs were cultured using high-glucose Dulbecco's modified Eagle medium (DMEM glucose $4.5 \mathrm{~g} / \mathrm{L}$ ) supplemented with $20 \%$ FBS (Thermo Fisher Scientific, Dreieich, Germany), 1\% Glutamax (Thermo Fisher Scientific, Dreieich, Germany), $5 \mu \mathrm{g} / \mathrm{mL}$ gentamicin (Thermo Fisher Scientific, Dreieich, Germany), in an incubator with a humidified atmosphere with $5 \% \mathrm{CO}_{2}$ at $37^{\circ} \mathrm{C}$. Single PEDOT:PSS scaffolds (P:P, COLL1, or COLL3) were cut into quarters. Three equally sized quarters were selected and placed into individual wells of a non-TC-treated plate. NCSCs were seeded at $1 \times 10^{5}$ cells/well/scaffold in $1 \mathrm{~mL}$ of the respective medium into a 24-well non-TC-treated cell culture plate. Cells were also seeded at the same seeding density ( $110^{5} /$ well $)$ in $1 \mathrm{~mL}$ as 2D control onto a 24-well TC-treated plate. Cells were cultured for a total of $48 \mathrm{~h}$ and the cell proliferation assay performed. Cell viability assays were performed for cells in both $2 \mathrm{D}$ and $3 \mathrm{D}$ cultures using the Cell Proliferation Kit II (XTT, Sigma-Aldrich, Taufkirchen, Germany) according to the manufacturer's instructions. Absorbance was measured at $490 \mathrm{~nm}$ and a reference wavelength of $650 \mathrm{~nm}$ on a Spectra Max iD3 plate reader (Molecular Devices, Wokingham, UK). Measurements were taken after $4 \mathrm{~h}$ of incubation. Values were corrected for the reference values $(650 \mathrm{~nm})$, as well as the appropriate PEDOT:PSS controls. For live cell monitoring, a suspension of NCSCs at P8 was membrane stained with PKH67 (PKH67 Green Fluorescent Cell Linker Kit for General Cell Membrane Labelling, Sigma-Aldrich, Taufkirchen, Germany) according to manufacturer's instructions. The scaffold was washed $3 \times$ with PBS prior to seeding. Images were collected every $15 \mathrm{~min}$ with bright field and the green fluorescent channel using the CQ1 by Yokogawa. Collected images were merged and videos were created. For osteogenic differentiation studies, scaffolds were seeded with $20 \mu \mathrm{L}$ of cell suspension using approximately 1500 cells/ $\mathrm{mm}^{3}$. To induce osteogenic differentiation, the medium was changed after 4 days to StemPro Osteocyte/Chondrocyte basal medium (Thermo Fisher Scientific, Dreieich, Germany) supplemented with StemPro Osteogenesis supplement (Thermo Fisher Scientific, Dreieich, Germany) according to the supplier instructions. Cultures were maintained at $37^{\circ} \mathrm{C}$ and $5 \% \mathrm{CO}_{2}$ for 21 days for Alizarin red S staining. Alizarin red $\mathrm{S}$ staining and its quantitative evaluation were performed according to a procedure previously described. ${ }^{[12,57]}$

\section{Statistical analysis}

Analysis of statistical differences was conducted using one-way ANOVA. Data were represented as averages \pm standard deviation $(* * * P<0.001)$.

\section{Supplementary material}

The supplementary material for this article can be found at https://doi.org/10.1557/mrc.2020.10.

\section{Acknowledgments}

D.I. would like to acknowledge the financial support by the H2020-MSCA-IF-2015 grant, "SMART-BONE" (GA No. 704175).

\section{References}

1. I.O. Foundation: Global Policy Initiatives 2015. https://www.iofbonehealth.org/global-policy-initiatives (cited January 9, 2020).

2. B. Antebi, G. Pelled, and D. Gazit: Stem cell therapy for osteoporosis. Curr. Osteoporos. Rep. 12, 41 (2014).

3. J. Kiernan, S. Hu, M.D. Grynpas, J.E. Davies, and W.L. Stanford: Systemic mesenchymal stromal cell transplantation prevents functional bone loss in a mouse model of age-related osteoporosis. Stem Cells Transl. Med. 5, 683 (2016).

4. A. Saito, K. Nagaishi, K. Iba, Y. Mizue, T. Chikenji, M. Otani, M. Nakano, K. Oyama, T. Yamashita, and M. Fujimiya: Umbilical cord extracts improve osteoporotic abnormalities of bone marrow-derived mesenchymal stem cells and promote their therapeutic effects on ovariectomised rats. Sci. Rep. 8, 1161 (2018).

5. Q. Wang, B. Zhao, C. Li, J.S. Rong, S.Q. Tao, and T.Z. Tao: Decreased proliferation ability and differentiation potential of mesenchymal stem cells of osteoporosis rat. Asian Pac. J. Trop. Med. 7, 358 (2014).

6. J. Tan, X. Xu, Z. Tong, J. Lin, Q. Yu, Y. Lin, and W. Kuang: Decreased osteogenesis of adult mesenchymal stem cells by reactive oxygen species under cyclic stretch: a possible mechanism of age related osteoporosis. Bone Res. 3, 15003 (2015).

7. L.C. Davies, H. Lonnies, M. Locke, B. Sundberg, K. Rosendahl, C. Gotherstrom, K. Le Blanc, and P. Stephens: Oral mucosal progenitor cells are potently immunosuppressive in a dose-independent manner. Stem Cells Dev. 21, 1478 (2012).

8. B. Kaltschmidt, C. Kaltschmidt, and D. Widera: Adult craniofacial stem cells: sources and relation to the neural crest. Stem Cell Rev. Rep. 8, 658 (2012).

9. S. Hauser, D. Widera, F. Qunneis, J. Muller, C. Zander, J. Greiner, C. Strauss, P. Luningschror, P. Heimann, H. Schwarze, J. Ebmeyer, H. Sudhoff, M.J. Arauzo-Bravo, B. Greber, H. Zaehres, H. Scholer, C. Kaltschmidt, and B. Kaltschmidt: Isolation of novel multipotent neural crest-derived stem cells from adult human inferior turbinate. Stem Cells Dev. 21, 742 (2012).

10. M. Schurmann, A. Wolff, D. Widera, S. Hauser, P. Heimann, A. Hutten, C. Kaltschmidt, and B. Kaltschmidt: Interaction of adult human neural crestderived stem cells with a nanoporous titanium surface is sufficient to induce their osteogenic differentiation. Stem Cell Res. 13, 98 (2014).

11. M. Schurmann, V. Brotzmann, M. Butow, J. Greiner, A. Hoving, C. Kaltschmidt, B. Kaltschmidt, and $H$. Sudhoff: Identification of a novel high yielding source of multipotent adult human neural crest-derived stem cells. Stem Cell Rev. Rep. 14, 277 (2018).

12. I. Azoidis, J. Metcalfe, J. Reynolds, S. Keeton, S.S. Hakki, J. Sheard, and D. Widera: Three-dimensional cell culture of human mesenchymal stem cells in nanofibrillar cellulose hydrogels. MRS Commun. 7, 458 (2017). 
13.J.F. Greiner, M. Gottschalk, N. Fokin, B. Buker, B.P. Kaltschmidt, A. Dreyer, T. Vordemvenne, C. Kaltschmidt, A. Hutten, and B. Kaltschmidt: Natural and synthetic nanopores directing osteogenic differentiation of human stem cells. Nanomedicine 17, 319 (2019).

14. M.A. Fernandez-Yague, S.A. Abbah, L. McNamara, D.I. Zeugolis, A. Pandit, and M.J. Biggs: Biomimetic approaches in bone tissue engineering: Integrating biological and physicomechanical strategies. Adv. Drug Deliv. Rev. 84, 1 (2015).

15. G.S. Hussey, J.L. Dziki, and S.F. Badylak: Extracellular matrix-based materials for regenerative medicine. Nat. Rev. Mater. 3, 159 (2018).

16. E. Fukada and I. Yasuda: On the piezoelectric effect of bone. J. Phys. Soc. Jpn 12, 1158 (1957)

17.F. Vasquez-Sancho, A. Abdollahi, D. Damjanovic, and G. Catalan: Flexoelectricity in bones. Adv. Mater. 30, 1705316 (2018).

18. C.A. Bassett, and R.O. Becker: Generation of electric potentials by bone in response to mechanical stress. Science 137, 1063 (1962).

19.B. Guo, and P.X. Ma: Conducting polymers for tissue engineering. Biomacromolecules 19, 1764 (2018).

20.J. Reynolds, B. Thompson, and T. Skotheim: Conjugated Polymers: Properties, Processing, and Applications (Taylor \& Francis, London, United Kingdom, 2019).

21. D. Khodagholy, J.N. Gelinas, Z. Zhao, M. Yeh, M. Long, J.D. Greenlee, W. Doyle, O. Devinsky, and G. Buzsaki: Organic electronics for highresolution electrocorticography of the human brain. Sci Adv. 2, e1601027 (2016).

22. S. Inal, A. Hama, M. Ferro, C. Pitsalidis, J. Oziat, I. Iandolo, A.M. Pappa, M. Huerta, D. Marchat, P. Mailley, and R.M. Owens: Conducting polymer scaffolds for hosting and monitoring 3D cell culture. Adv. Biosyst. 1, e1601027 (2017).

23. D. Iandolo, A. Ravichandran, X. Liu, F. Wen, J.K. Chan, M. Berggren, S.H. Teoh, and D.T. Simon: Development and characterization of organic electronic scaffolds for bone tissue engineering. Adv. Healthc. Mater. 5, 1505 (2016).

24. J. Rivnay, S. Inal, B.A. Collins, M. Sessolo, E. Stavrinidou, X. Strakosas, C. Tassone, D.M. Delongchamp, and G.G. Malliaras: Structural control of mixed ionic and electronic transport in conducting polymers. Nat. Commun. 7, 11287 (2016).

25. L.H. Jimison, S.A. Tria, D. Khodagholy, M. Gurfinkel, E. Lanzarini, A. Hama, G.G. Malliaras, and R.M. Owens: Measurement of barrier tissue integrity with an organic electrochemical transistor. Adv. Mater. 24, 5919 (2012).

26. D. Khodagholy, T. Doublet, P. Quilichini, M. Gurfinkel, P. Leleux, A. Ghestem, E. Ismailova, T. Herve, S. Sanaur, C. Bernard, and G.G. Malliaras: In vivo recordings of brain activity using organic transistors. Nat. Commun. 4, 1575 (2013).

27.A. Shahini, M. Yazdimamaghani, K.J. Walker, M.A. Eastman, H. Hatami-Marbini, B.J. Smith, J.L. Ricci, S.V. Madihally, D. Vashaee, and L. Tayebi: 3D conductive nanocomposite scaffold for bone tissue engineering. Int. J. Nanomedicine. 9, 167 (2014).

28. C. Pitsalidis, M.P. Ferro, D. Iandolo, L. Tzounis, S. Inal, and R.M. Owens: Transistor in a tube: A route to three-dimensional bioelectronics. Sci. Adv. 4, eaat4253 (2018).

29. M. Ramuz, A. Hama, M. Huerta, J. Rivnay, P. Leleux, and R.M. Owens: Combined optical and electronic sensing of epithelial cells using planar organic transistors. Adv. Mater. 26, 7083 (2014).

30. J. Rivnay, M. Ramuz, P. Leleux, A. Hama, M. Huerta, and R.M. Owens: Organic electrochemical transistors for cell-based impedance sensing. Appl. Phys. Lett. 106, 043301 (2015).

31. V.F. Curto, B. Marchiori, A. Hama, A.M. Pappa, M.P. Ferro, M. Braendlein, J. Rivnay, M. Fiocchi, G.G. Malliaras, M. Ramuz, and R.M. Owens: Organic transistor platform with integrated microfluidics for in-line multiparametric in vitro cell monitoring. Microsyst. Nanoeng. 3, 17028 (2017).

32. A.G. Guex, J.L. Puetzer, A. Armgarth, E. Littmann, E. Stavrinidou, E.P. Giannelis, G.G. Malliaras, and M.M. Stevens: Highly porous scaffolds of PEDOT:PSS for bone tissue engineering. Acta Biomater. 62, 91 (2017).

33. A.M. Ferreira, P. Gentile, V. Chiono, and G. Ciardelli: Collagen for bone tissue regeneration. Acta Biomater. 8, 3191 (2012).

34. A.A. Poundarik, A. Boskey, C. Gundberg, and D. Vashishth: Biomolecular regulation, composition and nanoarchitecture of bone mineral. Sci. Rep. 8, 1191 (2018)
35. A.C. Harley and L.J. Gibson: In vivo and in vitro applications of collagen-GAG scaffolds. Chem. Eng. J. 137, 102 (2008).

36.D.C. Chen, Y.L. Lai, S.Y. Lee, S.L. Hung, and H.L. Chen: Osteoblastic response to collagen scaffolds varied in freezing temperature and glutaraldehyde crosslinking. J. Biomed. Mater. Res. A 80, 399 (2007).

37.C.A. Mullen, M.G. Haugh, M.B. Schaffler, R.J. Majeska, and L.M. McNamara: Osteocyte differentiation is regulated by extracellular matrix stiffness and intercellular separation. J. Mech. Behav. Biomed. Mater. 28, 183 (2013).

38. J.G. Hardy, R.C. Cornelison, R.C. Sukhavasi, R.J. Saballos, P. Vu, D.L. Kaplan, and C.E. Schmidt: Electroactive tissue scaffolds with aligned pores as instructive platforms for biomimetic tissue engineering. Bioengineering (Basel) 2, 15 (2015).

39. R. Green and M.R. Abidian: Conducting polymers for neural prosthetic and neural interface applications. Adv. Mater. 27, 7620 (2015).

40. J.G. Hardy, R.C. Sukhavasi, D. Aguilar, M.K. Villancio-Wolter Jr. , D.J. Mouser, S.A. Geissler, L. Nguy, J.K. Chow, D.L. Kaplan, and C.E. Schmidt: Electrical stimulation of human mesenchymal stem cells on biomineralized conducting polymers enhances their differentiation towards osteogenic outcomes. J. Mater. Chem. B 3, 8059 (2015).

41. J. Pelto, M. Bjorninen, A. Palli, E. Talvitie, J. Hyttinen, B. Mannerstrom, R. Suuronen Seppanen, M. Kellomaki, S. Miettinen, and S. Haimi: Novel polypyrrole-coated polylactide scaffolds enhance adipose stem cell proliferation and early osteogenic differentiation. Tissue Eng. Part A 19, 882 (2013).

42. J.G. Hardy, S.A. Geissler, D. Aguilar Jr. , M.K. Villancio-Wolter, D.J. Mouser, R.C. Sukhavasi, R.C. Cornelison, L.W. Tien, R.C. Preda, R.S. Hayden, J.K. Chow, L. Nguy, D.L. Kaplan, and C.E. Schmidt: Instructive conductive 3D silk foam-based bone tissue scaffolds enable electrical stimulation of stem cells for enhanced osteogenic Differentiation. Macromol. Biosci. 15, 1490 (2015).

43. A.M. Wan, S. Inal, T. Williams, K. Wang, P. Leleux, L. Estevez, E.P. Giannelis, C. Fischbach, G.G. Malliaras, and D. Gourdon: 3D conducting polymer platforms for electrical control of protein conformation and cellular functions. J. Mater. Chem. B 3, 5040 (2015).

44. A. Berdichevski, M.A. Birch, and A.E. Markaki: Collagen scaffolds with tailored pore geometry for building three-dimensional vascular networks. Mater. Lett. 248, 93 (2019).

45. C.M. Murphy, M.G. Haugh, and F.J. O'Brien: The effect of mean pore size on cell attachment, proliferation and migration in collagenglycosaminoglycan scaffolds for bone tissue engineering. Biomaterials 31, 461 (2010).

46. A.J. Engler, S. Sen, H.L. Sweeney, and D.E. Discher: Matrix elasticity directs stem cell lineage specification. Cell 126, 677 (2006).

47. M.C. Varley, S. Neelakantan, T.W. Clyne, J. Dean, R.A. Brooks, and A.E. Markaki: Cell structure, stiffness and permeability of freeze-dried collagen scaffolds in dry and hydrated states. Acta Biomater. 33, 166 (2016).

48. G.S. Mandair, and M.D. Morris: Contributions of Raman spectroscopy to the understanding of bone strength. Bonekey Rep. 4, 620 (2015).

49. R. Dong, X. Yan, X. Pang, and S. Liu: Temperature-dependent Raman spectra of collagen and DNA. Spectrochim Acta A Mol. Biomol. Spectrosc. 60, 557 (2004).

50. S. Sakamoto, M. Okumura, Z. Zhao, and Y. Furukawa: Raman spectral changes of PEDOT-PSS in polymer light-emitting diodes upon operation. Chem. Phys. Lett. 412, 396 (2005)

51.X. Zhang, D. Chang, J. Liu, and Y. Luo: Conducting polymer aerogels from supercritical CO2 drying PEDOT-PSS hydrogels. J. Mater. Chem. 20, 5080 (2010).

52. J. Ouyang, C.W. Chu, F.C. Chen, Q. Xu, and Y. Yang: Polymer optoelectronic devices with high-conductivity poly(3,4-ethylenedioxythiophene) anodes. J. Macromol. Sci. - Pure Appl. Chem. 41A, 1497 (2004).

53.W.W. Chiu, J. Travas-Sejdic, R.P. Cooney, and G.A. Bowmaker: Spectroscopic and conductivity studies of doping in chemically synthesized poly(3,4-ethylenedioxythiophene). Synth. Met. 155, 80 (2005).

54. S. Garreau, G. Louarn, J.P. Buisson, G. Froyer, and S. Lefrant: In situ spectroelectrochemical Raman studies of Poly(3,4-ethylenedioxythiophene) (PEDT). Macromolecules 32, 6807 (1999).

55.Z. Zhang, G. Li, and B. Shi: Physicochemical properties of collagen, gelatin and collagen hydrolysate derived from bovine limed split wastes. $J$. Soc. Leather Technol. Chem. 90, 23 (2006) 
56. J.A. Uquillas, and 0. Akkus: Modeling the electromobility of type-I collagen molecules in the electrochemical fabrication of dense and aligned tissue constructs. Ann. Biomed. Eng. 40, 1641 (2012).

57. M.T. Zeuner, N.N. Didenko, D. Humphries, S. Stergiadis, T.M. Morash, K. Patel, W.D. Grimm, and D. Widera: Isolation and characterization of neural crest-derived stem cells from adult ovine palatal tissue. Front. Cell Dev. Biol. 6, 39 (2018).

58. D. Widera, C. Zander, M. Heidbreder, Y. Kasperek, T. Noll, O. Seitz, B. Saldamli, H. Sudhoff, R. Sader, C. Kaltschmidt, and B. Kaltschmidt: Adult palatum as a novel source of neural crest-related stem cells. Stem Cells 27, 1899 (2009).

59. J.F. Greiner, L.M. Grunwald, J. Muller, H. Sudhoff, D. Widera, C. Kaltschmidt, and B. Kaltschmidt: Culture bag systems for clinical applications of adult human neural crest-derived stem cells. Stem Cell Res. Ther. 5, 34 (2014).

60. Y.R. Shih, K.F. Tseng, H.Y. Lai, C.H. Lin, and O.K. Lee: Matrix stiffness regulation of integrin-mediated mechanotransduction during osteogenic differentiation of human mesenchymal stem cells. J. Bone Miner. Res. 26, 730 (2011).

61. C.A. Schneider, W.S. Rasband, and K.W. Eliceiri: NIH Image to ImageJ: 25 years of image analysis. Nat. Methods 9, 671 (2012). 